\title{
Wireless Head Gesture Controlled Robotic Wheel Chair for Physically Disable Persons
}

\author{
Shadman Mahmood Khan Pathan', Wasif Ahmed"1, Md. Masud Rana², Md. Shahjalal Tasin ${ }^{3}$, \\ Faisul Islam ${ }^{3}$, Anika Sultana ${ }^{3}$ \\ ${ }^{1}$ Department of Mechatronics, Rajshahi University of Engineering \& Technology, Rajshahi, Bangladesh \\ ${ }^{1}$ Department of Electrical \& Electronic Engineering, Rajshahi University of Engineering \& Technology, Rajshahi, Bangladesh \\ ${ }^{3}$ Department of EEE, Rajshahi University of Engineering \& Technology, Rajshahi, Bangladesh \\ Email:shadmanmahmood9@gmail.com,wasifahmed951@gmail.com, shahjalaltasin141014@ieee.org, \\ shahjalaltasin141015@ieee.org, Anikasultana@gmail.com,md.masud.rana.ruet@gmail.com
}

How to cite this paper: Pathan, S.M.K., Ahmed, W., Rana, Md.M., Tasin, Md.S., Islam, F. and Sultana, A. (2020) Wireless Head Gesture Controlled Robotic Wheel Chair for Physically Disable Persons. Journal of Sensor Technology, 10, 47-59. https://doi.org/10.4236/jst.2020.104004

Received: July 8, 2020

Accepted: December 28, 2020

Published: December 31, 2020

Copyright $\odot 2020$ by author(s) and Scientific Research Publishing Inc. This work is licensed under the Creative Commons Attribution International License (CC BY 4.0).

http://creativecommons.org/licenses/by/4.0/

(c) (i) Open Access

\begin{abstract}
A robotic wheelchair is assumed to be capable of doing tasks like navigation, obstacle detection, etc. using sensors and intelligence. The initial part of the work was development of a cap controlled wheelchair to test and verify the gesture operation. Following that, a real time operating wheelchair was developed consisting of mode changing option between joystick control mode and head gesture control mode as per as the user's requirement. The wheelchair consists of MPU6050 sensor, joystick module, RF module, battery, dc motor, toggle switch and Arduino. The movement of the head is detected by MPU6050 and the signal is transmitted to the microcontroller. Then the signal is processed by controller and motion of wheelchair is enabled for navigation. The wheelchair was capable of moving left, right, forward and backward direction. The speed of the wheelchair was $4.8 \mathrm{~km} / \mathrm{h}$ when tested. Design objective of the wheelchair included cost effectiveness without compromising safety, flexibility and mobility for the users.
\end{abstract}

\section{Keywords}

Head Gesture, Wheel Chair, Arduino, Motor Driver, Joystick Module

\section{Introduction}

Disability is a curse for a country particularly developing country. It is regarded as an embarrassment to the family the disabled people belongs to. This negligence of the society makes a bar between the persons with disabilities and the others in the society on normal economic, social and political activities in their families, communities, essential services and education. According to World 
Health Organization (WHO), "More than one billion people in the world live with some form of disability, of which nearly 200 million experience considerable difficulties in functioning" [1]. The amount of persons with disabilities is so high that it requires special consideration. WHO estimated the prevalence of the disabled person at $10.5 \%$ which is more common in the rural areas of the country [2]. The total rate of the physically disabled population is $27.5 \%$. In children below 18 years, the prevalence of disability is estimated at $6 \%$ and for the age group above 18 years, it is $14 \%$. It can be regarded as 3.4 million children with disabilities and 10.2 million adults with disabilities. And about $4.6 \%$ of people get disability due to spinal cord injury. To help physically challenged persons, there are many assistive technologies \& devices available [3]. Among the approaches to deal with disability issue, electric wheelchair is very much reliable. Mohammed Ismail. B et al. [3] developed a simple cost effective wheelchair with minimum assistance to the person suffering with Quadriplegia or Paraplegia. They proposed a microcontroller based control system by employing simple head gestures for directions like left, right, front, and back which is done by $\mathrm{Mi}$ cro-electromechanical systems (MEMS) technology. Rushikesh T. Bankar et al. [4] proposed a Field Programmable Gate Array (FPGA) based gesture recognition system on a wheelchair. The system was developed based on basically works on the principle of acceleration. The accelerometer is connected to ATMEGA328 Microcontroller which controls the direction of wheelchair. Shayban Nasif et al. [5] proposed a device which worked based on the Head Gesture Recognition technique using Acceleration sensor. Acceleration sensor is used for the head gesture recognition and RF (radio frequency) module is used for the smart wireless control. Jigmee Wangchuk et al. [6] designed a cost effective reliable robotic wheelchair which had capabilities of navigating, detecting obstacle and moving automatically by utilizing sensors which was controlled by head gestures. The head motion is calculating sensor value. Wheelchair avoids obstacles by ultrasonic sensor. Ankit Kunti et al. [7] proposed a micro-controller based smart wheelchair with different head gestures of the user. Ultrasonic sensor has been used as the medium of obstacle detector. G Azam et al. [8] proposed a voice controlled wheelchair which employs a voice recognition system which consists of microcontroller, microphone, voice recognition processor, motor control interface board to move the wheelchair. Users can operate this wheelchair by simply speaking in front of microphone. Kazi Ehsanul Karim et al. [9] proposed automated wheelchair with vertically adjustable seat which is microcontroller based. The fabrication required microcontroller, motor driver, DC motors and battery. Functions of the motor are controlled by microcontroller and motor driver by using Pulse Width Modulation (PWM). Trinayan Saharia et al. [10] proposed a joystick controlled wheelchair where microcontroller was used to process all the command. The controller sends digital signal to the motor driving Integrated Circuit (IC) and this IC control the movement of wheelchair. The input is sent from joystick which basically are the required commands needed to move the wheelchair. However, it is observed that most of the wheel chairs men- 
tioned are not accessible for mass people in the developing countries like Bangladesh.

In this paper, we are focusing on fabricating a wireless head gesture controlled smart wheelchair with joystick control. It will develop the self-assistive skill and they will be much more independent on controlling their movement. DC motor is used for the mobility of the wheelchair and sensor is used to sense the movement of head. We use simple sensors so that it can be cost effective \& there is a joystick module as a second control system for user interface. The motor we used are very powerful, but rest of the components are easy to access \& user friendly. This wheel chair can be controlled both head gesture \& joystick by switching their mode in a toggle switch.

\section{Methodology}

The model consists of transmitting part and receiving part. The block diagram of the proposed model consisting with transmitter and receiver section is shown in Figure 1. RF transmitter and RF receiver are used in this case. The MPU6050 sensor senses the rotation on $\mathrm{X}$-axis and $\mathrm{Y}$-axis. Based on that, the RF transmitter sends messages to RF receiver. If the sensed rotation is at positive $\mathrm{X}$-axis, the transmitter sends specific data. That means motor driver will drive the wheels at left. If the sensed rotation is at negative $\mathrm{X}$-axis, the transmitter sends another specific data. That means motor driver will drive the wheels at right. If the sensed rotation is at negative $\mathrm{Y}$-axis, the transmitter sends another value. That means motor driver will drive the wheels to forward. RF receiver is interfaced with Arduino mega2560 which accepts the messages from transmitter and sends them to Arduino mega2560. Arduino mainly converts these messages into American Standard Code for Information Interchange (ASCII) code and then this code is decoded. DC motors are driven by BTS7960 motor driver. Led acid battery is used for the purpose of mobility.

Prototype design of the wheelchair is discussed below.

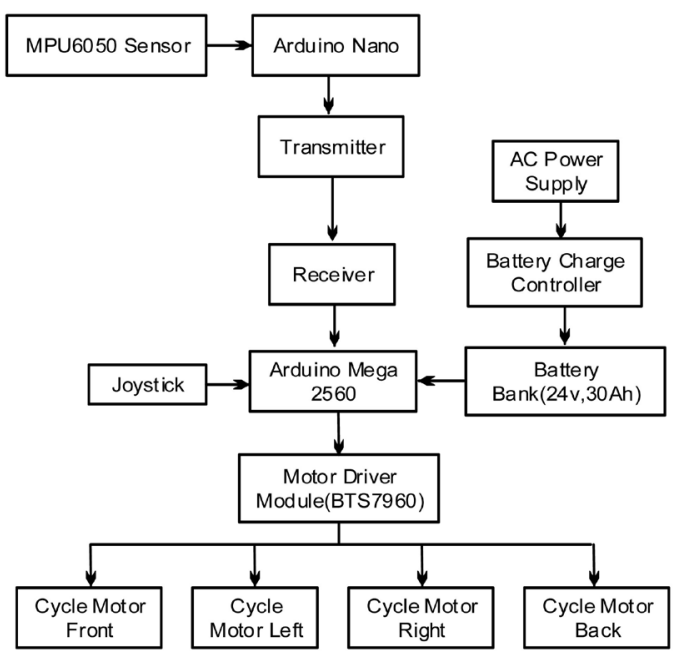

Figure 1. Block diagram of proposed wireless wheel chair. 


\section{Prototype Design of Wheelchair}

For better output, at first we made a prototype in which we mainly focus on the head gesture control system. In this prototype we use small dc motor, an Arduino, RF module \& L298N motor driver module. We have used RF module to reduce the use of wire and avoid complex circuitry.

In Figure 2, the transmitting section with Arduino Nano is shown. The sensor is also connected in the transmitter. The Vcc \& GND pin of both sensor \& transmitter is connected with the $+5 \mathrm{~V} \&$ GND pin of Arduino and D12 is used for transmitter. A4 \& A5 is used for the sensor data.

In Figure 3, we see that there are a receiver, a motor driver (L298N), a battery, two dc gear motor \& an Arduino mega2560. Here is a $12 \mathrm{~V}$ supply which is given into $12 \mathrm{~V}$ port of motor driver module. The enable \& input pin is connected into PWM section of the arduino. Both the output is connected into the motor. Moreover arduino powered from the motor driver $5 \mathrm{~V}$ output port. There is a battery which gives power to the whole circuit (Table 1).

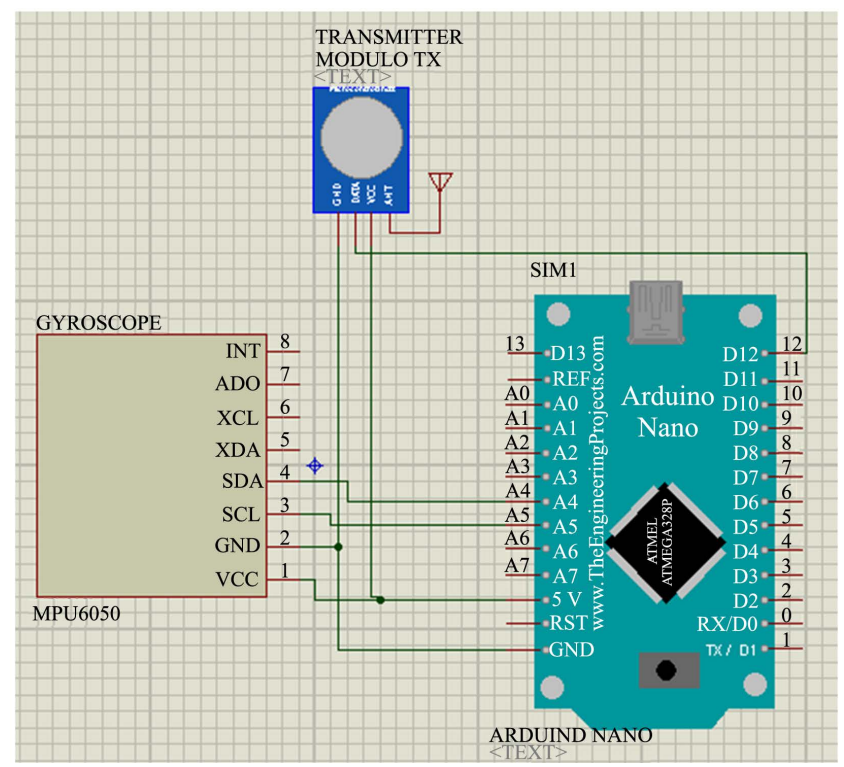

Figure 2. Transmitting section circuit for prototype \& real model.

Table 1. List of components for prototype design.

\begin{tabular}{cc}
\hline SL No. & ITEMS \\
\hline 1 & Wheel (1")-No.-2 \\
2 & Ball Caster-No.-1 \\
3 & Battery-No.-2 \\
4 & Gear Motor (12V)-No.-2 \\
5 & L298 Motor Driver-No.-1 \\
6 & MPU6050 Sensor module-No.-1 \\
7 & RF Module-No.-1 \\
8 & Arduino mega2560-No.-1 \\
9 & Arduino Nano-No.-1
\end{tabular}




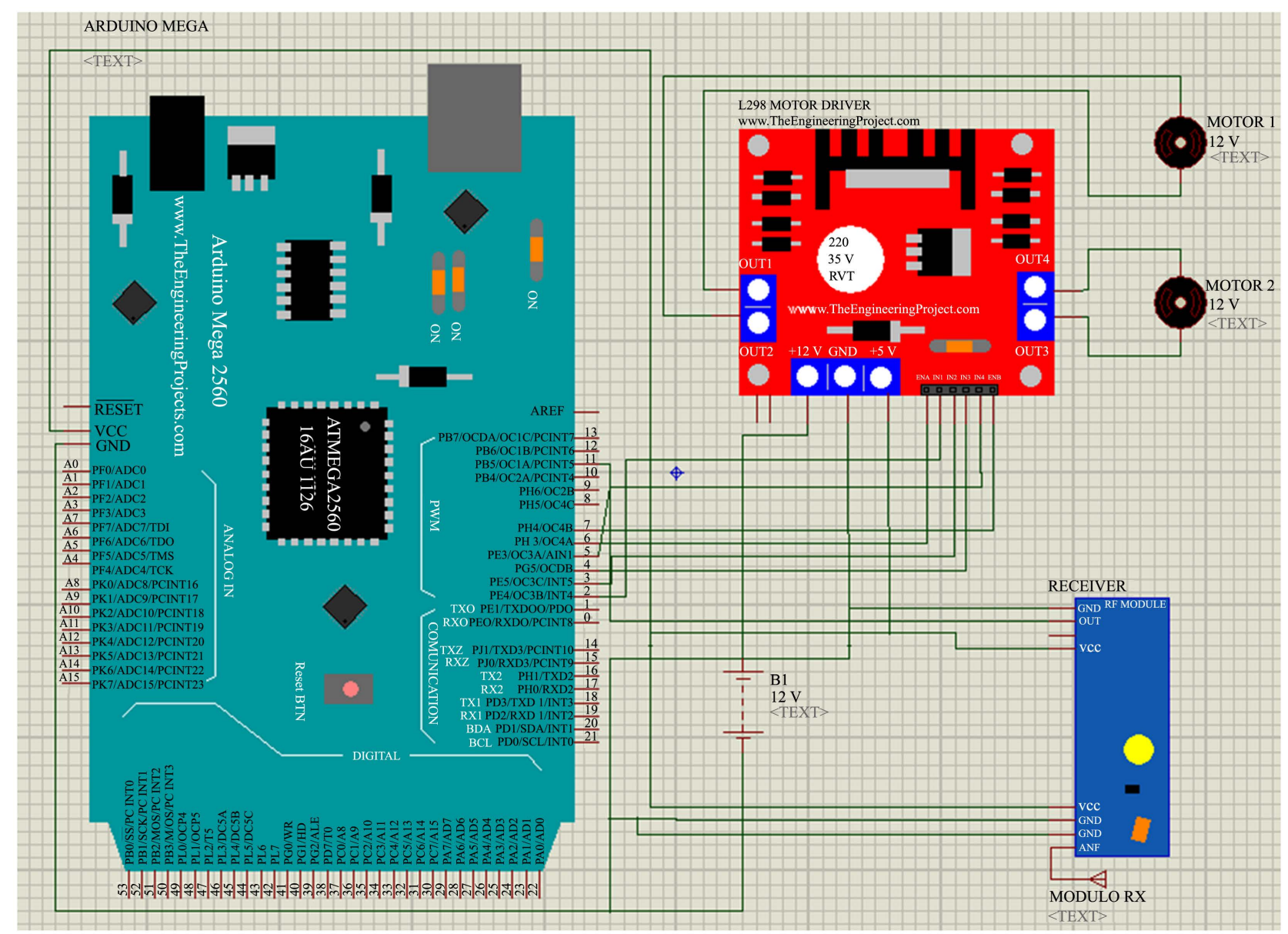

Figure 3. Receiving section circuit for prototype.

A lithium polymer (LiPo) battery is a rechargeable battery that is used as the power source to drive the wheelchair. These batteries provide higher specific energy than other lithium battery. LiPo battery of $900 \mathrm{mAh}$ is used to get the best power solution. There are two gear motors that drive the left and right wheel autonomously. This 12 V DC gear motor can draw 200 - $600 \mathrm{~mA}$ current and provides rotational speed of 30 RPM. It has the benefits of providing high torque and low noise. Hence, the performance of wheelchair depends on the high efficiency of motor. The $\mathrm{L} 298 \mathrm{~N}$ is a dual H-Bridge motor driver which allows speed and direction control of two DC motors at the same time. The module can drive DC motors that have voltages between 5 and $35 \mathrm{~V}$ with a peak current up to $2 \mathrm{~A}$. The maximum power consumption is $20 \mathrm{~W}$. MPU6050 sensor module is known as 6 axis Motion tracking device. It basically consists of 3-axis gyroscope, 3-axis accelerometer and Digital Motion Processor all in small package. Basically, the RF modules are $433 \mathrm{MHz} \mathrm{RF}$ transmitter and receiver modules. When logic one is sent, carrier is fully on to about $4.5 \mathrm{~mA}$ with a 3 volts power supply. The data is sent serially from the transmitter which is received by tuned receiver. The operating voltage of transmitter is $2-12 \mathrm{~V}$ and the operating frequency is $433 \mathrm{MHz}$. it can transmits any signal within the range of $300-500$ 
$\mathrm{m}$. Input signal is always at TTL logic level. The operating voltage for receiver is $5.0 \mathrm{VDC}+0.5 \mathrm{~V}$ and the working current is below $2.5 \mathrm{~mA}$. The operating frequency is $433 \mathrm{MHz}$. The Arduino Mega 2560 is a microcontroller which is board based on the ATmega2560. It has 54 digital input/output pins of which 15 can be used as PWM outputs. 16 analog inputs, 4 UART, a $16 \mathrm{MHz}$ crystal oscillator, a USB connection, a power jack, an ICSP header and a reset button. The Arduino Nano is a small, complete, and breadboard-friendly board based on the ATmega328P (Arduino Nano 3.x). It has more or less the same functionality of the Arduino Uno, but in a different package. The receiver section of the prototype wheel chair is shown in Figure 4.

The receiver section consists of Receiver, Arduino Mega, L298N motor and wheel. The receiving signal will be analyzed by Arduino Mega which is connected to L298N motor driver. According to signal, the motor driver connected to the wheels will control the four directions which are moving left, right, forward and backward (Figure 5).

In the prototype design, an arduino nano is connected with the sensor \& transmitter. The arduino is powered by a battery and the whole system is mounted in the cap. There is a delay which makes the cap more effective from random tilt of head. That means if anyone move their head randomly the gesture won't work. To work the gesture, one must keep the head to the desired direction for a certain time.

\section{Hardware Implementation}

After completing the prototype design and tested properly, we implemented the real wheel chair. But we use some upgraded motor, motor driver module and more powerful battery for the real structure. We use stainless steel pipe to make the frame. The back wheel we used in this structure is cycle wheel. Caster wheel is used as front wheel. The schematic diagram is shown in Figure 6.

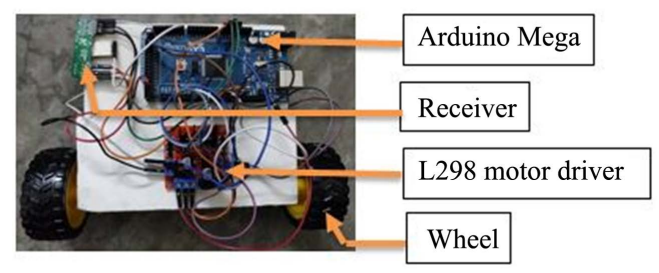

Figure 4. Prototype design (Receiving section).

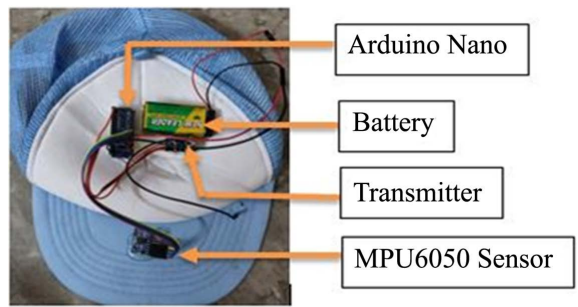

Figure 5. Cap (Transmitting section). 


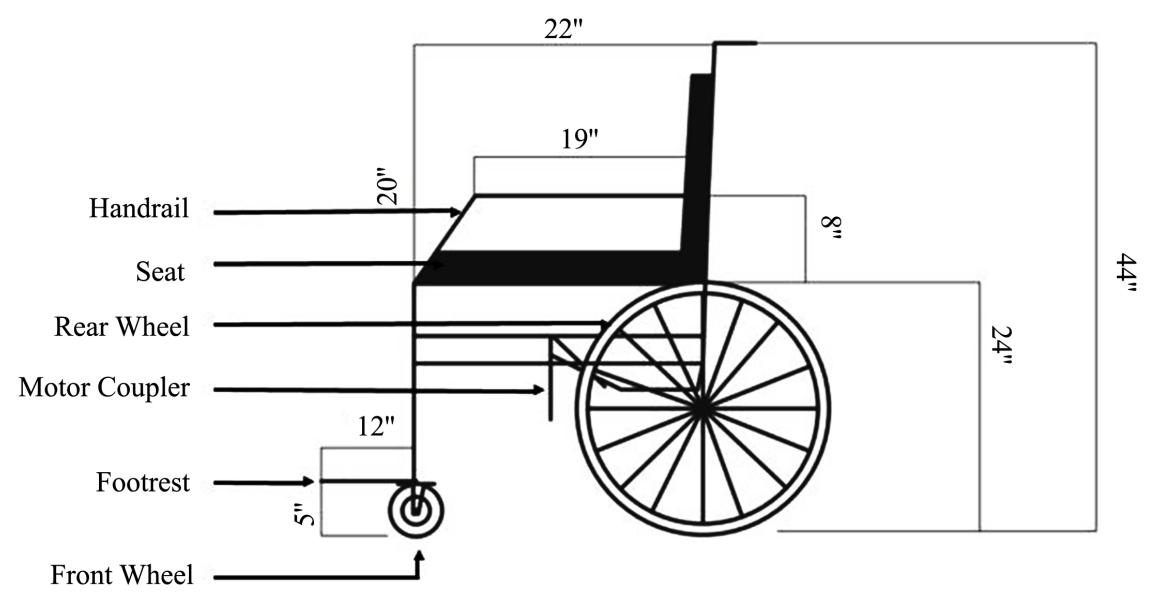

Figure 6. Structure design of real wheel chair.

This is the structural layout of the proposed model. It actually represents the hardware implementation of the project. This figure shows the side view of the wheelchair. There are two rear wheels with large diameter of 24 " which are attached at the back of the wheelchair. Two small front wheels are also there with a diameter of 5 " which are in left side and right side of the chair as rear wheels. At the joining point of each front wheel, a footboard of 12 " is attached for comfort of the user. On the both side of the chair there is a handrail of 19" which are in height of 8 " from the rear wheel. Handrails are for comfort as well as the attachment of joystick. Two parallel horizontal lines are shown which seems gone through the rear wheel on both side of the chair. Upper line represents the 22" connecting pipe between front and back main structural pipe with whom coupling plate of the motor will be attached. The bottom line represents the 22 " supporting pipe of the wheelchair for better stability. A vertical pipe is also connected on both sides for the same reason. Bottom of the wheelchair there is a case for the battery. From the seat to the top of the wheelchair it is about 20 " tall. The total height of the wheelchair from the ground is $44 "$. Receiver section is shown in Figure 7.

In Figure 7, we see that there is joystick module, motor driver (2), Receiver, Motor, Battery \& a toggle switch. Two motor driver modules are connected with the digital PWM pin of the Arduino. There is also a connection of the $24 \mathrm{~V}$ battery in both motor drivers. Both the motors are also connected into the motor driver. To give supply in the Arduino we use a small battery. For the transmitter we use same circuit that used for the prototype design. We use the similar components that used for prototype designing in the real wheel chair, but we use upgraded dc motor, motor driver module, battery, wheel \& joystick module is also added. The list of components used for real wheel chair is shown in Table 2.

To understand the algorithm of the project there is a flow chart which describes the whole algorithm. The flow chart is shown in Figure 8.

Here we see that, the whole algorithm is mainly depended into the toggle switch mode. If it is high, then the joystick control algorithm is enabling. But if 
Table 2. List of components used for real wheel chair.

\begin{tabular}{cc}
\hline SL No. & ITEMS \\
\hline 1 & Wheel (24")-No.-2 \\
2 & Caster wheel (5")-No.-2 \\
3 & Stainless Steel frame \\
4 & Led Acid Battery (12V)-No.-2 \\
5 & DC Motor (24V, 250 Watt)-No.-2 \\
6 & BTS7960 Motor driver-No.-2 \\
7 & MPU6050 Sensor module-No.-1 \\
8 & RF Module-No.-1 \\
9 & Joystick module-No.-1 \\
10 & Arduino mega2560-No.-1 \\
11 & Arduino Nano-No.-1 \\
12 & Flash light-No.-1 \\
\hline
\end{tabular}

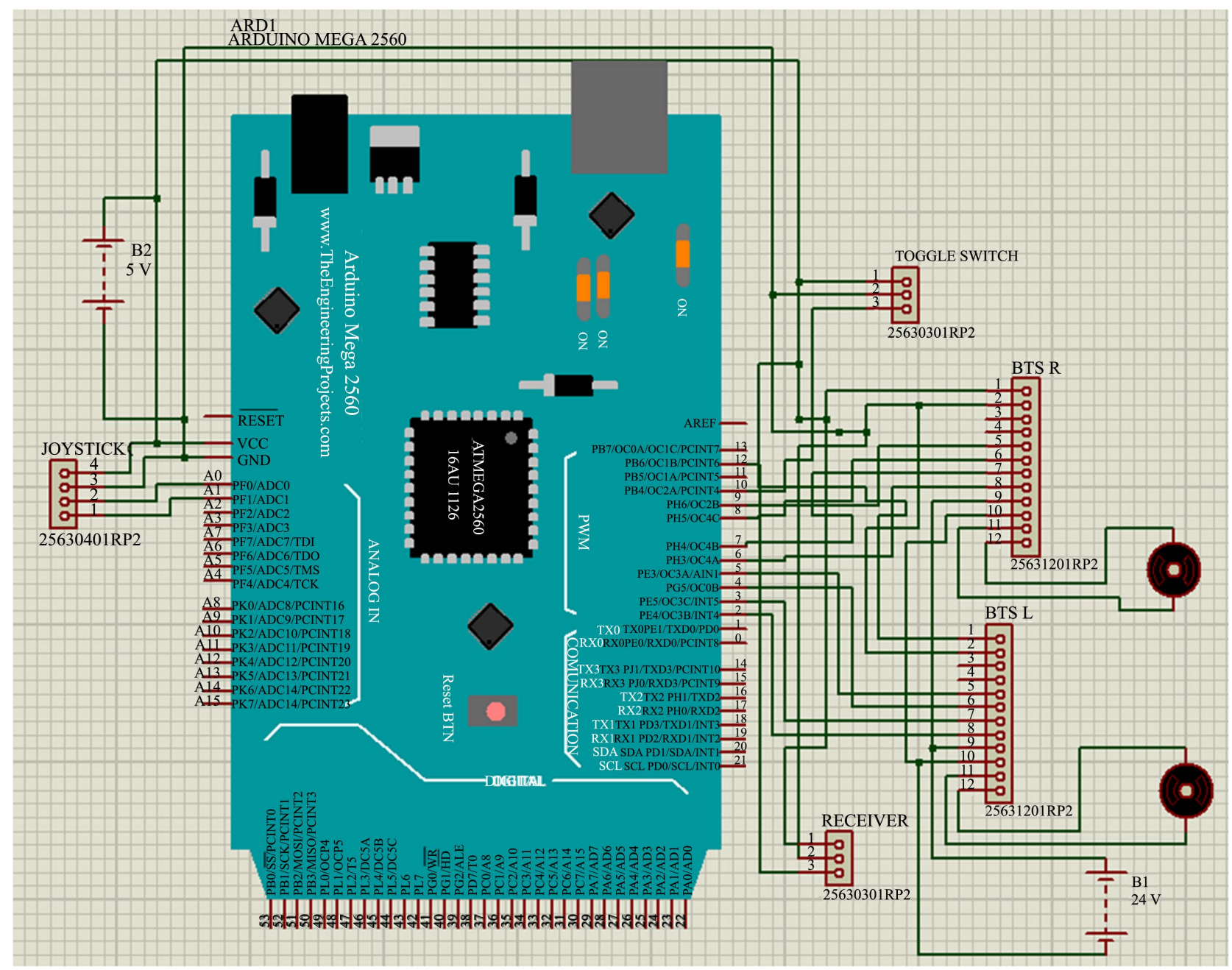

Figure 7. Schematic diagram of the receiving circuit for real wheel chair. 


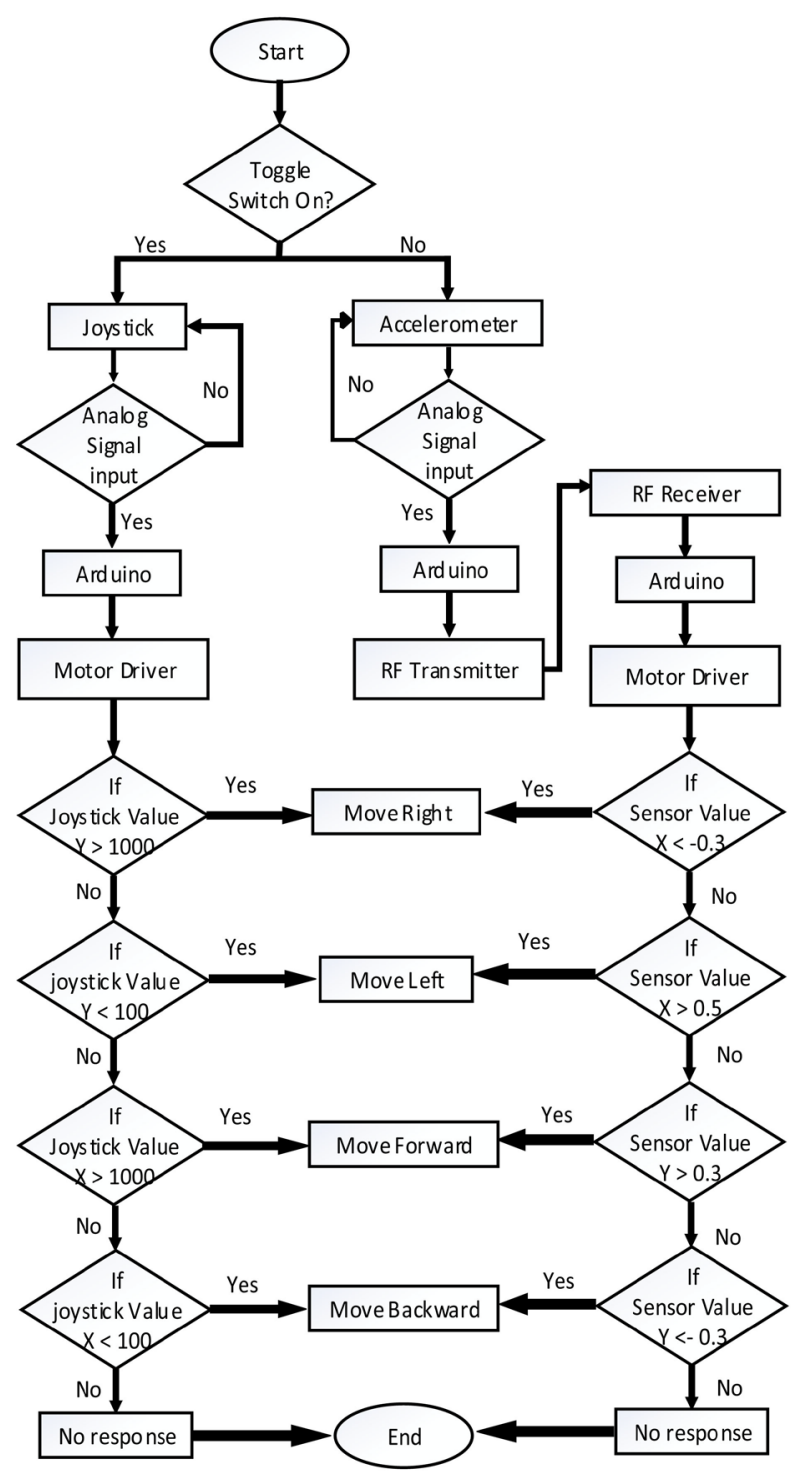

Figure 8. Flow chart of the algorithm.

the toggle switch is off, that means the mode is low, then head gesture control system is on. Then depending on the sensor values, the wheel chair will work. Based on the algorithm, the hardware implementation of the real wheel chair is shown in Figure 9.

Figure 9 shows the real model of a wheel chair. After the fabrication of the wheelchair, we measured the curvature of wheelchair which is $27 \mathrm{inch}$. We tested the wheelchair in indoor environment and the result was satisfactory. The cap was mounted on a single user and the movement of head controls the direction of the wheelchair. Depending on the MPU 6050 sensor values, the wheel chair moves on various direction is shown in Table 3 . If the $\mathrm{X}$-axis value is $<-0.3$, the chair moves in the right direction and for $\mathrm{Y}$-axis value $>0.3$ moves in the forward direction. Similarly, for the $\mathrm{X}$-axis value is $>0.5$ moves in the left and for $\mathrm{Y}$-axis value $<-0.3$ moves on backward direction. 


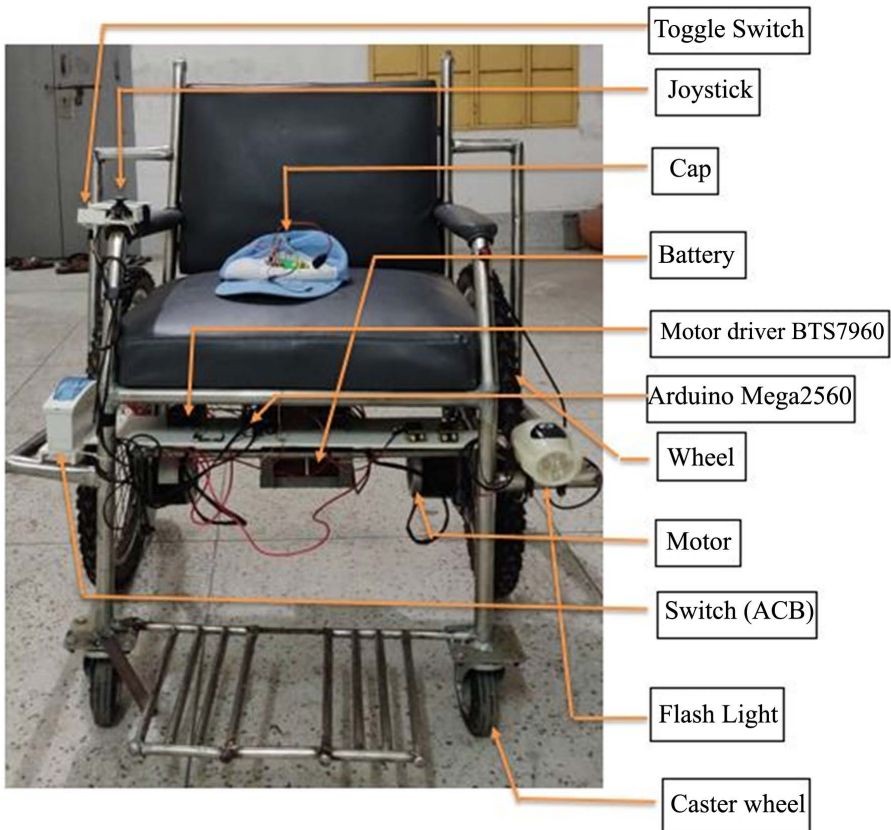

Figure 9. Hardware implementation of the wheel chair.

Table 3. MPU6050 sensor value \& direction.

\begin{tabular}{cc}
\hline Sensor value & Direction \\
\hline $\mathrm{X}<-0.3$ & Right \\
$\mathrm{X}>0.5$ & Left \\
$\mathrm{Y}>0.3$ & Forward \\
$\mathrm{Y}<-0.3$ & Backward \\
\hline
\end{tabular}

The output of left, right, forward and backward direction with real image is shown Figure 10.

After interfacing the joystick module with the microcontroller, we can expect four outputs from the wheelchair. As 2-axis joystick has been used, we can get right, left, forward and backward directions for different values in the $\mathrm{x}$-axis and $y$-axis. From the table given above, we can see that for negative values in the $\mathrm{x}$-axis, the wheelchair will move to the right. For positive values in the $\mathrm{x}$-axis, the wheelchair will move to the left. For positive values in the y-axis, the wheelchair will move backward. And for negative values in the y-axis, the wheelchair will move forward. The joystick value is shown in Table 4.

For smoothness we gradually increase the speed of the motor. After taking several reading we find out that the average time required to reach in its maximum speed which we provided is 3.8 seconds. We also calculate the velocity by taking a fixed distance of two (2) meter.

$$
V=s / t
$$

where $s=2 \mathrm{~m} \& t=1.5 \mathrm{~s}$ (average).

So, $V=4.8 \mathrm{~km} / \mathrm{h}$. 


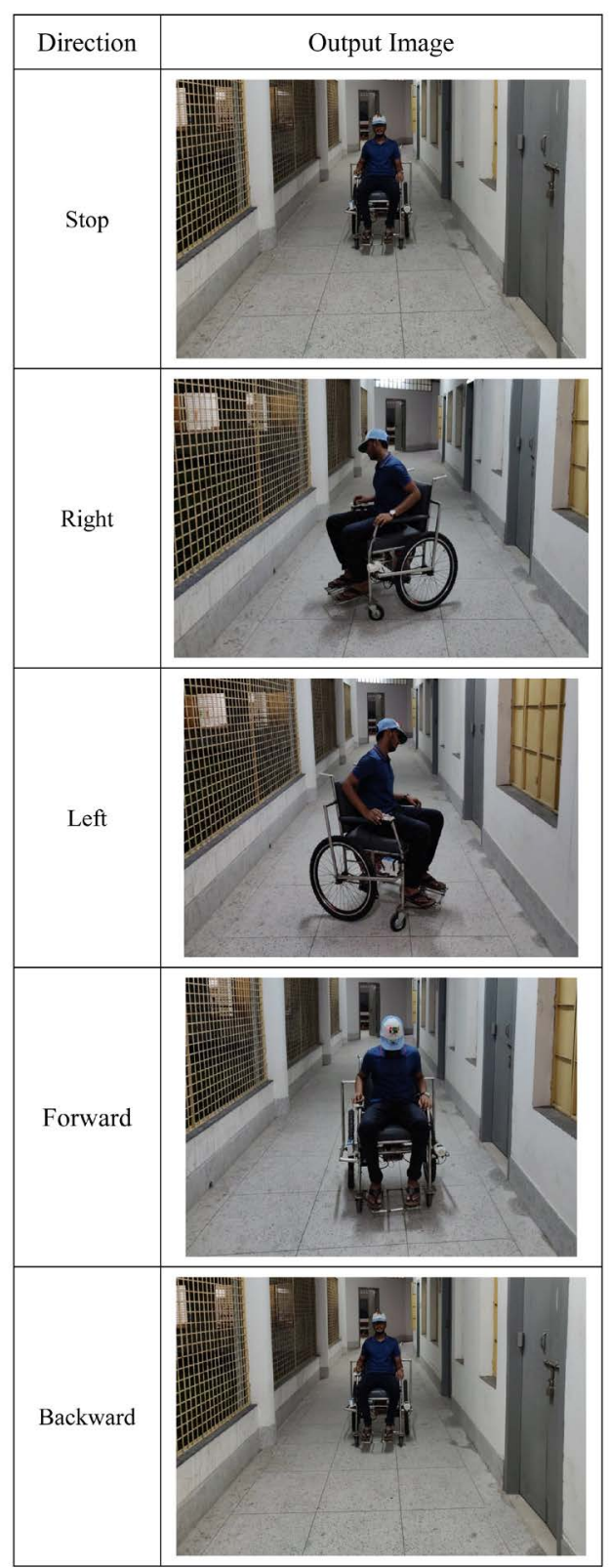

Figure 10. Operational direction with output images.

Table 4. Joystick value \& direction.

\begin{tabular}{cc}
\hline Joystick value & Direction \\
\hline $\mathrm{Y}>1000, \mathrm{X}<600 \& \mathrm{X}>400$ & Right \\
$\mathrm{Y}<100, \mathrm{X}<600 \& \mathrm{X}>400$ & Left \\
$\mathrm{X}>1000, \mathrm{Y}<600 \& \mathrm{Y}>400$ & Forward \\
$\mathrm{X}<100, \mathrm{Y}<600 \& \mathrm{Y}>400$ & Backward \\
\hline
\end{tabular}

The cost analysis of the wheelchair is shown in Table 5. Total cost of wheelchair is BDT 41,000 that is equivalent to USD 500 only excluding the labour cost which is very cheap compared to the chair available in the market. 
Table 5. Cost analysis.

\begin{tabular}{ccc}
\hline Serial no. & Item description & Cost (USD) \\
\hline 01 & Frame (SS pipe 1", SS pipe 0.75", sheet metal 0.5") & 100.40 \\
02 & Cycle wheel (2 pcs) & 41.34 \\
03 & Caster wheel (2 pcs) & 17.72 \\
04 & LED acid battery (2 pcs) & 98.03 \\
05 & Cycle motor (2 pcs) & 144.10 \\
06 & Arduino Mega (1 pcs) & 9.45 \\
07 & Arduino NANO & 4.72 \\
08 & Motor driver (4 pcs) & 37.80 \\
09 & PCB design & 2.36 \\
10 & Connecting wire & 3.54 \\
11 & MPU6050 Sensor & 4.72 \\
12 & RF module & 3.54 \\
& Total & 467.72 \\
\hline
\end{tabular}

\section{Conclusion}

In this paper, we develop a wheel chair which is cost effective for a disabled person to lead his or her day to day life. We use a simple sensor that makes it cost effective \& there is a joystick module as a second control system for user interface. The motor we used are very powerful, but rest of the components are easy to access \& user friendly. This wheel chair can be controlled both head gesture \& joystick by switching their mode in a toggle switch. Before implementing the real wheel chair, we develop a prototype cap controlled wheelchair and tested the operation and to work gesture properly, one must keep the head to the desired direction for a certain time. After that, real time Robotic Wheel Chair is developed that has mode changing options which are joystick control mode and head gesture control mode as per as the user's requirement. The controller processes the signal and enables the motion of wheelchair for its navigation. The implemented wheelchair functioned properly and moved left, right, backward and forward direction according to the program given into microcontroller. The speed of the wheel chair is $4.8 \mathrm{~km} / \mathrm{h}$. Moreover, the wheelchair is designed in a cost-effective way but ensures safety, flexibility, and mobility for the users.

\section{Acknowledgement}

The authors would like to show their gratitude to University Grant Commission (UGC), Bangladesh for granting financial support to this work.

\section{Conflicts of Interest}

The authors declare no conflicts of interest regarding the publication of this paper. 


\section{References}

[1] Siddharth, P.D. and Deshpande, S. (2016) Embedded System Design for Real-Time Interaction with Smart Wheelchair. 2016 Symposium on Colossal Data Analysis and Networking, Indore, 18-19 March 2016, 1-4. https://doi.org/10.1109/CDAN.2016.7570917

[2] Srivastava, P. and Thakur, R. (2014) A Novel Head Gesture Recognition Based Control for Intelligent Wheelchairs. International Journal of Research in Electrical \& Electronics Engineering, 2, 10-17.

[3] Ismail, M., Fouzan Ishaqui, B.S., Karim Khan, A. and Abdul Quadeer, M. (2014) Head Gesture Based Control of Wheelchair for a Paralysed Person in an Indoor Environment. International Journal of Innovative Research in Electrical, Electronics, Instrumentation and Control, 2, 2079-2083. https://doi.org/10.17148/IJIREEICE.2014.0210006

[4] Bankar, R.T. and Salankar, S.S. (2015) Implementation of an Intelligent Head Gesture Recognition System. International Journal of Innovative Science and Modern Engineering, 3, 2319-6386.

[5] Nasif, S. and Khan, M.A.G. (2017) Wireless Head Gesture Controlled Wheel Chair for Disable Persons. 2017 IEEE Region 10 Humanitarian Technology Conference, Dhaka, 21-23 December 2017, 156-161. https://doi.org/10.1109/R10-HTC.2017.8288928

[6] Machangpa, J.W. and Chingtham, T.S. (2018) Head Gesture Controlled Wheelchair for Quadriplegic Patients. Procedia Computer Science, 132, 342-351.

https://doi.org/10.1016/j.procs.2018.05.189

[7] Kunti, A., Chouhan, V., Singh, K., Yadav, A.R., Yadav, I. and Pankaj, D. (2018) Head-Motion Controlled Wheel Chair Direction Using ATMega328p Microcontroller. International Journal of Advanced Research in Computer and Communication Engineering, 7, 61-65.

[8] Azam, G. and Islam, M.T. (2015) Design and Fabrication of a Voice Controlled Wheelchair for Physically Disabled People. International Conference on Physics for Sustainable Development \& Technology, Chittagong, 19-20 August 2015, 81-90.

[9] Karim, K.E., Ahmed, H. and Nahiyan, H. (2014) Design and Simulation of an Automated Wheelchair with Vertically Adjustable Seat. International Conference on Mechanical Industrial \& Energy Engineering, Khulna, 25-26 December, 2014, 1-6.

[10] Saharia, T. and Bauri, J. (2017) Joystick Controlled Wheelchair. International Research Journal of Engineering and Technology, 4, 235-237. 